AC

$$
\begin{aligned}
& \text { IPNO. TH } 93.72 \\
& \text { SW } 9410
\end{aligned}
$$

\title{
ON THE COHERENCE BETWEEN HIGH-ENERGY TOTAL CROSS-SECTION DATA WHEN COMPARED WITH GENERAL PRINCIPLES
}

\author{
Pierre GAURON and Basarab NICOLESCU
}

Division de Physique Théorique*, IPN, 91406 Orsay Cedex and LPTPE, Université Pierre et Marie Curie, 4 Place Jussieu, 75252 Paris Cedex 05, France

\footnotetext{
* Unité de Recherche des Universités Paris 11 et Paris 6 Associée au CNRS
} 


\begin{abstract}
We perform an essentially model - independent study of the internal coherence between high-energy total cross-section data by using classes of functions satisfying general principles. Our study is practically independent of the $\rho$-parameter values. Our general analysis, made without any fit, reveals certain inconsistencies in the existing set of high-energy data. Some of these inconsistencies are eliminated by giving up arbitrary assumptions sometimes made in "fitology". We conclude that the $\ln ^{2} s$ increase of total cross-sections at high energies is clearly favoured when compared with the other possible behaviours.
\end{abstract}


Hadronic total cross-sections $\sigma_{T}$ are one of the most elementary and, at the same time, the most fundamental notions in particle physics. It is therefore natural that their theoretical study has received much attention in the past, from the historical paper of Heisenberg on the $\ln ^{2} s$ behaviour of $\sigma_{T}$ [1] to the derivation of one of the most rigorous results of hadron physics - the Froissart-Martin asymptotic bound [2].

The discovery, in the early 1970's, of the increase of $\sigma_{T}^{p p}$ in the ISR energy range [3] reactivated interest in the study of total cross-sections. This discovery was anticipated by the prediction based on the phenomenological study of cosmic-ray data [4] and it was confirmed by later experiments performed at ISR both in $p p$ and $\vec{p} p$ scattering [5].

On the theoretical level [6] two facts were striking, from the very moment of the discovery of increasing total cross-sections : 1) the increase is perfectly compatible with the $\ln ^{2} s$ behaviour of $\left.\sigma_{T} ; 2\right)$ the new term, responsible for the $\ln ^{2} s$ increase of $\sigma_{T}$ acts as a small correction in the ISR energy range when compared with the usual Regge terms. Therefore, the minimum in $\sigma_{T}^{p p}$ or $\sigma_{T}^{\bar{p} p}$ - of the order of $40 \mathrm{mb}$ - appeared as a new scale in hadron physics, set by low energy contributions and giving a concrete meaning to the realm of asymptotic energies : if $\sigma_{T}^{p p}$ or $\sigma_{T}^{\bar{p} p}$ would be of the order of 500 $\mathrm{mb}$, then we could really speak of an asymptotic regime. For the moment, ISR appears as a transition region between low energies and high energies.

A huge number of theoretical and phenomenological articles followed this experimental discovery. However no review was written untill now covering simultaneously the four main theoretical approaches of $\sigma_{T}$ : asymptotic theorem - inspired models, QCD inspired models, eikonal formalism and Reggeon Field Theory. In any case, because of its theoretical implications, the $\ln ^{2} s$ behaviour was in the past the most favoured possibility.

The advent of CERN $S \bar{p} p S$ and Tevatron machines allowed us to penetrate deeper in the high-energy range. However here the situation became quite confused. While the UA4 $\sigma_{T}$ datum [7] remains compatible with the $\ln ^{2} s$ increase of $\sigma_{T}$, two contradictory results were obtained at the Tevatron : the E710 result [8] seems to indicate a deviation 
towards a ln $s$ behaviour [9] while the recent CDF result [10], $8 \mathrm{mb}$ higher than the E710 result, is still compatible with the $\ln ^{2} s$ behaviour.

It is therefore important to question the coherence between high-energy $\sigma_{T}$ data when compared with general theoretical requirements.

In order to perform this study we will not resort to any fitology. Fitology - the science of fits, based on $\chi^{2}$ minimization - has, of course, many virtues but has also, in our opinion, a major defect : the details of minimization are often crucial in getting the main physical conclusions. Let us give just one example in order to express what we have in mind. Say that in an experiment (like E710) the raw data are $d N / d y(y$ is a direction perpendicular to the beam) and not $d N / d t$. To each fixed y corresponds an interval in $t$. One then extracts $d N / d t$ by using an exponential $\exp (B t)$ form, with $B=$ const. One finally performs a $\chi^{2}$ minimization on the $d N / d t$ data so extracted and one deduces that the slope of the differential cross-section is constant. It is clear in this example that the physical conclusion was already contained in the details of the minimization. Many other similar examples could be given.

We will perform instead an exercise in "experimental mathematics", without any fit. We consider classes of functions respecting the general principles and modelling in fact an infinite number of possible scattering amplitudes. We then inject as little as possible experimental input in order to extract as much as possible theoretical predictions. We finally compare the theoretical numbers with the experimental bounds and suggest that there are possible internal self-contradictions of the high energy $\sigma_{T}$ data (i.e. ISR, CERN $S \bar{p} p S$, Tevatron and cosmic-ray data).

We first consider the following class of functions supposed to be valid for both $p p$ and $\bar{p} p$ scattering (no-Odderon case) :

$$
\sigma_{T}=C_{1}+C_{2} \ln ^{\gamma}\left(\frac{s}{s_{+}}\right)
$$

where $\gamma$ is left free (of course, the Froissart-Martin bound [2] requires $\gamma \leq 2$ ). 
We are namely interested in the increase of $\sigma_{T}$ in the range $\left(s_{1}, s_{2}\right)$, with $s_{2}>s_{1}$ :

$$
\left.\Delta \sigma(\gamma)\right|_{s_{1}} ^{s_{2}}=C_{2}\left[\ln ^{\gamma}\left(\frac{s_{2}}{s_{+}}\right)-\ln ^{\gamma}\left(\frac{s_{1}}{s_{+}}\right)\right],
$$

the parameter $C_{1}$ being so eliminated from our study.

Let us fix for the moment the scale factor $s_{+}$to be

$$
s_{+}=1 \mathrm{GeV}^{2}
$$

in order to get the Amaldi form*[11], popular amongst experimentalists and recently used in fits performed by the UA4/2 collaboration [12]. Of course, there is no physical reason to fix $s_{+}$to such a value. Moreover, from the mathematical point a view such a procedure is simply wrong : the 3-parameter form of eq. (2) cannot be reduced to a 2-parameter form without an arbitrary supplementary condition such as (3). However let us accept for the moment the condition (3) in order to compare with the results of ref. [12]. The case of arbitrary $s_{+}$will be considered later.

The procedure is the following : 1) we first take as experimental input the increase of $\sigma_{T}$ between CERN $S \bar{p} p S$ and Tevatron energies ; 2) we then compute, at arbitrary fixed $\gamma$, the value of $C_{2}$ extracted from eq. (2);3) we finally compute $\left.\Delta \sigma(\gamma)\right|_{s_{1}} ^{s_{2}}$ by using eq. (2), for the cases $\left(\sqrt{s_{1}}=62.3 \mathrm{GeV}, \sqrt{s_{2}}=541 \mathrm{GeV}\right)$ and $\left(\sqrt{s_{1}}=1.8 \mathrm{TeV}\right.$, $\sqrt{s_{2}}=30 \mathrm{TeV}$ ) and compare with the experimental data.

It has to be noted that, in the CDF case, the values of $\rho$ and $\sigma_{T}$ are not given separately, only the value $\left(1+\rho^{2}\right) \sigma_{T}$ being measured. We will assume for the moment $\rho=0.135$ in agreement with the UA4/2 [13] and E710 [8] values. This corresponds to $\sigma_{T}=62.2 \pm 1.5 \mathrm{mb}$ at CERN $S \bar{p} p S$ energy $\sqrt{s}=541 \mathrm{GeV}[12]$.

In fact our study has the advantage of being practically independent of the assumed value of $\rho$. Of course, at fixed $\mathrm{s}$, the value of $\sigma_{T}$ is sensitive to the assumed value of $\rho$, when the experimental $\left(1+\rho^{2}\right) \sigma_{T}$ value is imposed : for example, when

* The familiar Regge terms, present in the Amaldi form, are practically negligible in our energy range. 
$\rho$ increases from 0.135 to 0.2 , the $\sigma_{T}$ decreases by $1.3 \mathrm{mb}$ at $\sqrt{s}=541 \mathrm{GeV}$ and by $1.7 \mathrm{mb}$ at $\sqrt{s}=1.8 \mathrm{TeV}$. However this variation at fixed $\mathrm{s}$ is practically cancelled when the difference of $\sigma_{T}$ between $s_{1}$ and $s_{2}$ is considered : when $\rho$ varies, $\sigma_{T}\left(s_{1}\right)$ and $\sigma_{T}\left(s_{2}\right)$ both decrease (or increase) by almost the same amount. This is in fact the reason why we chose to study $\left.\Delta \sigma(\gamma)\right|_{s_{1}} ^{s_{2}}$ and not $\sigma_{T}\left(s_{1}\right)$ and $\sigma_{T}\left(s_{2}\right)$ themselves.

There are two experimental inputs at fixed $\gamma:\left.\Delta \sigma^{\exp }(\gamma)\right|_{\text {UA } / 2} ^{C D F}$ and $\left.\Delta \sigma^{\exp }(\gamma)\right|_{U \wedge 4 / 2} ^{E 710}$, corresponding to the two different experimental results at $\sqrt{s}=1.8$ $\mathrm{TeV}[8,10]$. The results of our procedure are shown in figs. 1 and 2 , where only the range $0.5 \leq \gamma \leq 2.5$ is explicitely exhibited. In these figures the lower band corresponds to the allowed experimental increase of $\sigma_{T}$ between $\sqrt{s}=62.3 \mathrm{GeV}[14]$ and $\sqrt{s}=541$ $\mathrm{GeV}[12]$, while the upper band is, in fact, the lower bound on the increase of $\sigma_{T}$ between $\sqrt{s}=1.8 \mathrm{TeV}$ and $\sqrt{s}=30 \mathrm{TeV}$, the AKENO best fit [15] being considered in the cosmic-ray range of energies. The central line in the upper band is affected by an indicative error of $\pm 10 \mathrm{mb}$, typical for the cosmic-ray energy range.

From the fig. 1 it is seen that, in the case of the $\left.\Delta \sigma^{\exp }(\gamma)\right|_{V A 4 / 2} ^{C D F}$ input, the theoretical points corresponding to the increase of $\sigma_{T}$ between ISR and CERN $S \bar{p} p S$ energies marginally meet the allowed experimental range only for values of $\gamma$ corresponding to the violation of the Froissart-Martin bound : $2 \lesssim \gamma \lesssim 2.5$. The lower bound on the increase of $\sigma_{T}$ between Tevatron (TEV) and cosmic-ray (CR) energies is respected for all $\gamma$ values in the considered range $(0.5,2.5)$.

The situation in fig. 2, corresponding to the $\left.\Delta \sigma^{\exp }(\gamma)\right|_{U A 4 / 2} ^{E 710}$ input, is somewhat reversed. The theoretical points corresponding to $\left.\Delta \sigma(\gamma)\right|_{\text {ISR }} ^{C E R S_{P p S}}$ meet the allowed experimental strip for all values of $\gamma$, with a slight preference for the range $0.7 \leqslant \gamma \leqslant 1.4$ (to which the magical value $\gamma=1$ prefered in ref. 9 belongs). However the theoretical points are systematically lower than the lower experimental bound on $\left.\Delta \sigma(\gamma)\right|_{\text {TEV }} ^{C R}$.

It is now interesting to compare our results with the detailed 8-parameter fit of $\sigma_{T}$ in the energy range $5 \lesssim s \lesssim 541 \mathrm{GeV}$ performed by the UA4/2 collaboration [12]. The relevant parameter in the context of our discussion is $\gamma$. The value of $\gamma$ extracted from 
the fit of ref. [12] is 2.25 affected by a positive error of 0.35 and by a negative error of 0.31. This result is perfectly compatible with our result shown in fig. 1. (It is amusing to note that, in ref. [12], the predicted value of $\sigma_{T}$ at $\sqrt{s}=1.8 \mathrm{TeV}$ is $76.5 \pm 2.3 \mathrm{mb}$, in between the E710 and CDF experimental values : $\simeq 72 \mathrm{mb}$ and $\simeq 80 \mathrm{mb}$, respectively). However, the violation of the Froissart-Martin bound is just a mathematical artefact of the arbitrary condition (3), as we will show in the following.

Let us now study the effect of ruling out the condition (3), which obviously introduces (see eq. 1) a strong and artificial correlation between the parameters $\gamma$ and $C_{2}$ and therefore leads to doubtful predictions at high energies. The above procedure can be easily extended in the case of arbitrary $s_{+}$by systematically exploring, for fixed $\gamma$, a given range of $s_{+}$, say $10 \leq s_{+} \leq 500 \mathrm{GeV}^{2}$. It has to be noted that $\gamma=1$ is a special case : the quantity $\left.\Delta \sigma(\gamma=1)\right|_{s_{1}} ^{s_{2}}$ defined by eq. (2) is obviously independent of $s_{+}$. However, for $\gamma \neq 1$, the dependence on $s_{+}$has interesting consequences, especially when $\gamma$ is far from the special case $\gamma=1$ :

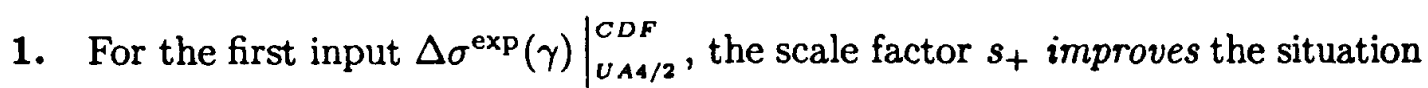
described in fig. 1. Namely, for $\gamma>1$ the quantity $\left.\Delta \sigma(\gamma)\right|_{I S R} ^{C E R N} S_{\bar{p} p S}$ decreases and for $\gamma \simeq 2$ the theoretical points meet the experimental allowed region (while $\left.\Delta \sigma(\gamma)\right|_{T E V} ^{C R}$ increases, the agreement with the experimental bound being kept). For example, for

$$
\gamma=2 \quad \text { and } \quad s_{+}=240 \mathrm{GeV}^{2}
$$

we get

$$
C_{2}=0.47 \pm 0.07 \mathrm{mb}
$$

and, therefore,

$$
\left.\Delta \sigma(\gamma=2)\right|_{I S R} ^{C E R N S_{\tilde{p} p S}}=20.08 \pm 2.97 \mathrm{mb}
$$

and

$$
\left.\Delta \sigma(\gamma=2)\right|_{T E V} ^{C R}=65.13 \pm 9.65 \mathrm{mb}
$$

in agreement with the experimental constraints (see fig. 1).

2. Paradoxically enough, for the second input $\left.\Delta \sigma^{\exp }(\gamma)\right|_{U A 4 / 2} ^{E 710}$, the scale factor $s_{+}$worsens the situation described in fig. $2:\left.\Delta \sigma(\gamma)\right|_{I S R} ^{C E R N S s_{p} S}$ increases for $\gamma<1$ and 
decreases for $\gamma>1$ and meets the experimental band for $\gamma$-values around 1. The agreement of $\left.\Delta \sigma(\gamma)\right|_{T E V} ^{C R}$ with the experimental bound is somewhat improved for $2 \lesssim \gamma \leqslant 2.5$ but, for this range of $\gamma$, the agreement of $\left.\Delta \sigma(\gamma)\right|_{\text {ISR }} ^{C E R N} S_{p p S}$ with the experimental constraint is destroyed.

In conclusion, our study, involving the general class of functions (1-2), singles out the CDF data (when compared with the E710 data) and the physical value $\gamma=2$. The agreement with the Froissart-Martin bound, in the CDF case, is restored by considering the scale factor $s_{+} \neq 1 \mathrm{GeV}^{2}$. In this context, the CDF data appears as one of the most significant experimental results obtained in the last years.

One might ask if our conclusions remain valid for cases which are not covered by our class of functions, such as the case $\sigma_{T} \rightarrow$ const. as $s \rightarrow \infty$.

In order to answer this question we consider the class of functions proposed by Bourrely and Martin [16], which were published before the Tevatron data were measured :

$$
F^{+}(s)=i s \frac{A+B\left(\ln \frac{s}{s_{0}}-i \frac{\pi}{2}\right)^{2}}{1+C\left(\ln \frac{s}{s_{0}}-i \frac{\pi}{2}\right)^{2}}
$$

i.e.

$$
\sigma_{T}=\frac{\left[A+B\left(\ln ^{2} \frac{s}{s_{0}}-\frac{\pi^{2}}{4}\right)\right]\left[1+C\left(\ln ^{2} \frac{s}{s_{0}}-\frac{\pi^{2}}{4}\right)\right]+\pi^{2} B C \ln ^{2} \frac{s}{s_{0}}}{\left[1+C\left(\ln ^{2} \frac{s}{s_{0}}-\frac{\pi^{2}}{4}\right)\right]^{2}+\pi^{2} C^{2} \ln ^{2} \frac{s}{s_{0}}}
$$

For $C=0$, one recovers our class of functions (1) via the trivial equivalence formulae :

$$
B=C_{2}, \quad s_{0}=s_{+}, \quad A=C_{1}+C_{2} \frac{\pi^{2}}{4}
$$

However, for $C \neq 0$ one gets the important physical case of asymptotically constant total cross-sections. Before studying this last case it is important to note that the solution I of Bourrely-Martin (BM) :

$$
C=0, \quad B=0.43 \mathrm{mb}, \quad s_{0}=243.6 \mathrm{GeV}^{2}, \quad A=41.695 \mathrm{mb}
$$


is numerically equivalent to our case (4-5). Both these cases predict that the asymptotic regime of $\sigma_{T}$ is established at $\sqrt{s} \simeq 10^{8} \mathrm{GeV}$. (Of course, in our case, the numerical value of $A$ was not needed to be known and, in order to extract $B$ and $s_{0}$, we did not perform any kind of fit). Therefore all the previous discussion, in the case of our first experimental input, remains valid. The solution BM I is in agreement with the new CDF datum [10] : Bourrely and Martin predicted that $\sigma_{T}=81.1 \mathrm{mb}$ at $\sqrt{s}=2 \mathrm{TeV}$ [16].

Our previous very simple procedure can not be directly applied to the BM class of functions because of the obvious complexity introduced by the form (9). It can be nevertheless adapted to this case and interesting questions can be answered in a still simple and general way.

An important remark is that the physically distinct solutions I and II of Bourrely and Martin still have a common feature : the numerical values of the parameters $A$ and $s_{0}$ are practically the same in both cases. Namely, the solution BM II corresponds to the following numerical values:

$$
C=6.10^{-3}, \quad B=0.815 \mathrm{mb}, \quad s_{0}=277.7 \mathrm{GeV}^{2}, \quad A=41.824 \mathrm{mb} .
$$

It is seen from (11) and (12) that the fit with $C \neq 0$ induces a brutal change only in the value of the $B$ parameter. This remark raises a lot of interesting questions.

Let us first start from the solution BM I and formulate the following question : what happens if we allow for $C \neq 0$, but keeping fixed the values of $\mathrm{B}, s_{0}$ and $\mathrm{A}$ as given by (11). The answer is shown in fig. $3 \mathrm{a}$, where we directly plotted $\sigma_{T}$ in terms of C. The perturbation in $\mathrm{C}$ around the solution BM I shows that the agreement of the theoretical points with the experimental constraints (in particular, with the CDF data) still can be kept for $C \neq 0$ (in the range $0<C \leqslant 3.10^{-4}$ ). This conclusion might be surprising, because $C \neq 0$ case corresponds to a radically distinct case (asymptotically constant cross-sections) from the $C=0$ one (asymptotic cross-sections growing like $\ln ^{2} s$ ), but this sudden transition is realized by a very small change of the value of $\mathrm{C}$ and could be experimentally detected only at ultra-high energies. 
A similar question can be formulated in the case of solution BM II : what happens if we perform a perturbation in $\mathrm{C}$ by keeping fixed the values of $\mathrm{B}, s_{0}$ and $\mathrm{A}$ as given by (12). The answer is shown in fig. $3 \mathrm{~b}$. A new feature appears: it is the UA4 datum which imposes an important constraint. When $\mathrm{C}$ increases in the range $5.10^{-3} \leqslant C \leqslant 7 \cdot 10^{-3}$, the agreement with the UA4 datum is kept, but $\sigma_{T}$ rapidly jumps from the CDF value to the E710 value at $\sqrt{s}=1.8 \mathrm{TeV}$, while the agreement with the AKENO best-fit lower bound in the cosmic-ray region is not improved. In other words, it is difficult in this case to choose between E710 and CDF data if one neglects the cosmic-ray data.

More sophisticated analysis can be performed by keeping fixed the parameters $\mathbf{A}$ and $s_{0}$ and by using a simultaneous perturbation in $\mathrm{B}$ and $\mathrm{C}$ around the solution BM I and BM II, respectively. We made such a search but no new information was obtained when compared with the above analysis.

In conclusion, our study, which can be considered as a simple exercise in "experimental mathematics", shows how important theoretical conclusions can be drawn from a very poor experimental input without appealing to any kind of fitology. Moreover, most of our results are practically independent of the $\rho$ values in this energy region.

We show, by using two classes of functions satisfying general principles and which model a practically infinite number of functions, that the $\ln ^{2} s$ increase of total crosssections at high energies is clearly favoured. However a $\ln s$ increase is not totally excluded if one does not insist on experimental constraints involving cosmic-ray data. On other side, our general study reveals some internal inconsistencies in the existing set of high-energy data. Some of these inconsistencies can be eliminated if one does give up some arbitrary assumptions made in the fitology.

However the ultimate judge is always the experimental data. The publication of the luminosity dependent $\sigma_{T}$ obtained in the already performed UA4/2 experiment would bring interesting information for the clarification of the present situation. Without waiting for the important (but far away) experimental results from RHIC and LHC machines, a new high-precision experiment at the Tevatron is badly needed. The 
theoretical interest of such experiments is clear : striking trends of a spectacular simplification appear already in the maximum complexity region of hadron physics - the elastic and diffractive scattering.

Acknowledgements. We thank Drs. Maurice Haguenauer, Shekhar Shukla and Jorge Velasco for useful discussions on experimental data and Prof. Elliot Leader for a careful reading of the manuscript.

\section{References}

[1] W. Heisenberg, Z. Phys. 133 (1952) 65.

[2] M. Froissart, Phys. Rev. 123 (1961) 1053;

A. Martin, Phys. Rev. 129 (1963) 1432; Nuovo Cimento 42A (1966) 930.

[3] U. Amaldi et al., Phys. Lett. B44 (1973) 112;

S.R. Amendolia et al., Phys. Lett. B44 (1979) 119; Nuovo Cimento 17A (1973) 735.

[4] G.B. Yodh, Y. Pal and J.S. Trefil, Phys. Rev. Lett. 28 (1972) 1005.

[5] See for example :

R210 Collab., G. Carboni et al., Nucl. Phys. B254 (1985) 697.

R211 Collab., N.A. Amos et al., Nucl. Phys. B262 (1985) 689.

[6] E. Leader and U. Maor, Phys. Lett. B43 (1973) 505.

[7] UA4 Collab., M. Bozzo et al., Phys. Lett. B147 (1984) 392.

[8] E710 Collab., N.A. Amos et al., Phys. Lett. B243 (1990) 158; Phys. Rev. Lett. 68 (1992) 2433;

S. Sadr, talk at the Vth International Conference on the Elastic and Diffractive Scattering (Vth Blois Workshop), Providence, USA., June 1993, to be published in the proceedings of this conference.

[9] M.M. Block and A.R. White, Phys. Lett. B273 (1991) 145.

[10] CDF Collab., F. Abe et al., FERMILAB-Pub-93/234-E preprint, submitted to Phys. Rev D.

[11] U. Amaldi et al., Phys. Lett. B66 (1977) 390.

[12] UA4/2 Collab., C. Augier et al., Phys. Lett. B315 (1993) 503.

[13] UA4/2 Collab., C. Augier et al., Phys. Lett. B316 (1993) 448.

[14] R211 Collab., see ref. [5].

[15] M. Honda et al., Phys. Rev. Lett. 70 (1993) 525.

[16] C. Bourrely and A. Martin, Proc. of the ECFA-CERN Workshop, CERN, Service d'Information Scientifique (1984), p. 233. 


\section{FIGURE CAPTIONS}

Fig. 1 The increase $\Delta \sigma$ of $\sigma_{T}$ in terms of $\gamma$ when the CDF data [10] are used as input at $\sqrt{s}=1.8 \mathrm{TeV}$.

Fig. 2 The increase $\Delta \sigma$ of $\sigma_{T}$ in terms of $\gamma$ when the E710 data [8] are used as input at $\sqrt{s}=1.8 \mathrm{TeV}$.

Fig. 3a Perturbation in C of the solution I BM [16].

Fig. 3b Perturbation in $\mathrm{C}$ of the solution II BM [16]. 


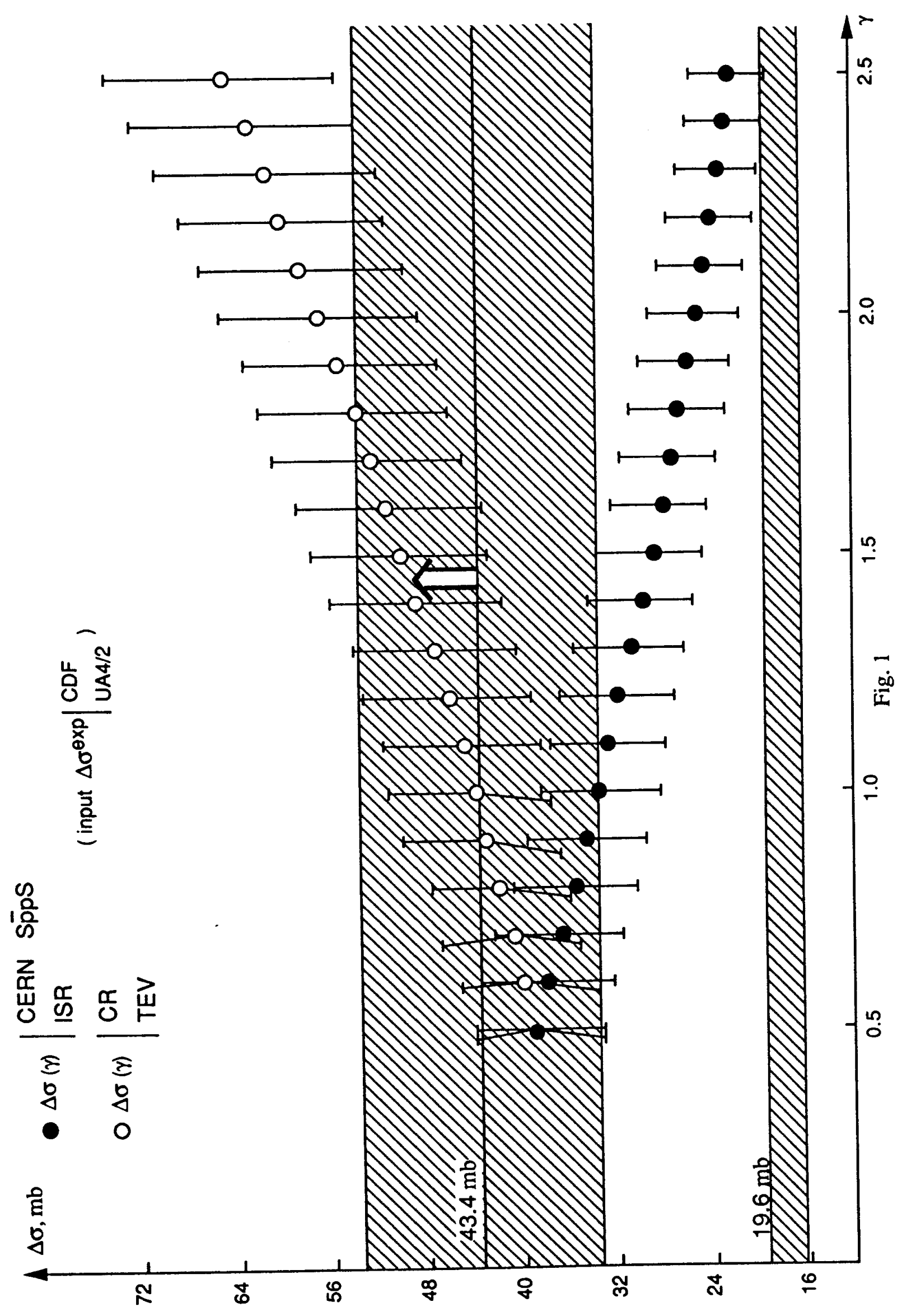




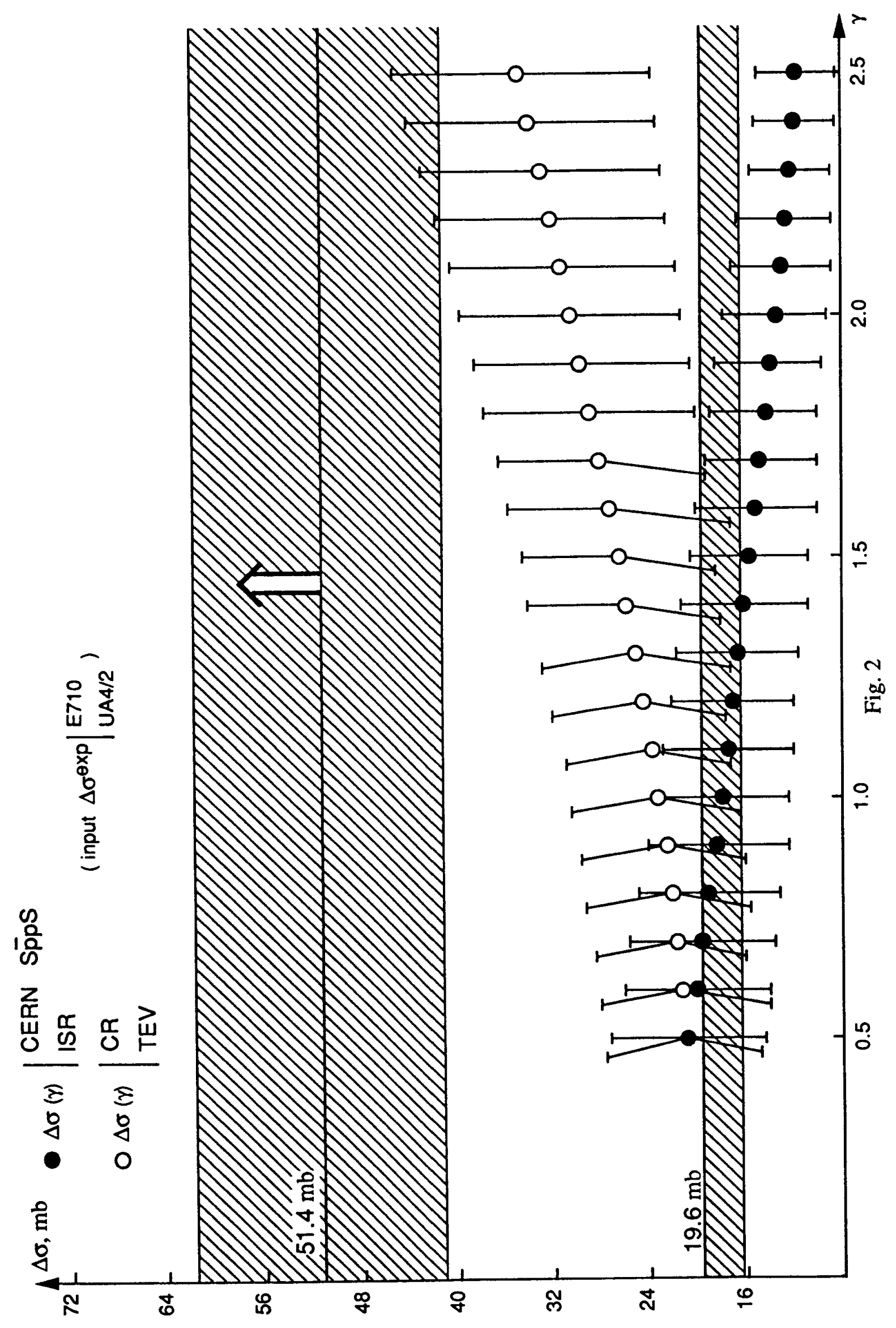




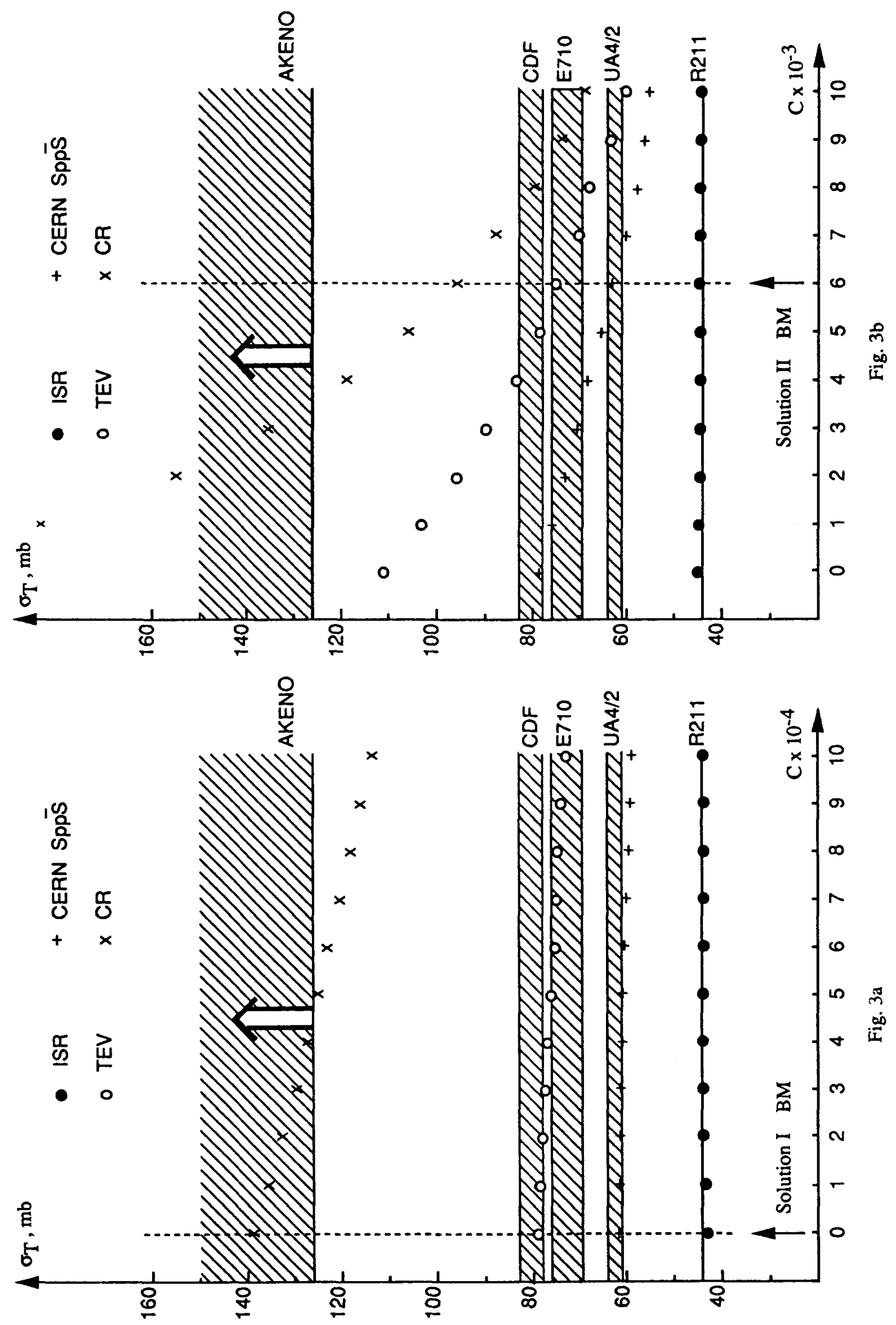

